\title{
Influência dos níveis de concentrado sobre os cortes comerciais, os constituintes não-carcaça e os componentes da perna de cordeiros confinados ${ }^{1}$
}

\section{Rossana Herculano Clementino ${ }^{2}$, Wandrick Hauss de Sousa ${ }^{3}$, Ariosvaldo Nunes de Medeiros $^{4}$, Maria das Graças Gomes Cunha ${ }^{5}$, Severino Gonzaga Neto ${ }^{4}$, Francisco Fernando Ramos de Carvalho ${ }^{6}$, Maria Andréa Borges Cavalcante ${ }^{7}$}

\author{
${ }^{1}$ Parte da Dissertação apresentada pela primeira autora como exigência para conclusão do Curso de Mestrado em Zootecnia - UFPB, \\ financiada pelo CNPq. \\ ${ }^{2}$ Mestre em Zootecnia e doutoranda em Zootecnia do PDIZ - UFC. \\ ${ }^{3}$ Orientador, EMEPA. \\ ${ }^{4}$ DZ/CCA/UFPB. \\ 5 EMEPA-PB. \\ ${ }^{6}$ Departamento de Zootecnia - UFRPE. Bolsista do CNPq \\ ${ }^{7}$ Bolsista DCR/DZ/UFC, Fortaleza-CE
}

\begin{abstract}
RESUMO - Um experimento foi realizado com o objetivo de determinar a influência dos níveis de concentrado sobre o peso e o rendimento dos cortes e dos constituintes não-carcaça e a composição tecidual da perna de cordeiros mestiços de Dorper terminados em confinamento ou mestiços de Dorper x Santa Inês. Foram utilizados 32 cordeiros (não-castrados) $1 / 2$ Dorper $x$ 1/2 Santa Inês (peso médio inicial de 17,1 kg) alimentados com dietas contendo 30, 45, 60 e $75 \%$ de concentrado. Os cordeiros foram distribuídos em um delineamento inteiramente casualizado com quatro tratamentos e oito repetições. Os animais foram mantidos em confinamento durante 43 dias. Após o abate, as carcaças foram resfriadas por 24 horas. Foram registrados os pesos e as porcentagens dos cortes da meia-carcaça e as porcentagens de osso, músculos e tecido adiposo, avaliadas por meio de dissecação da perna. Houve efeito significativo linear crescente dos níveis de concentrado sobre os pesos de pescoço, paleta, costilhar, lombo e perna. Verificou-se também efeito significativo linear decrescente sobre os rendimentos dos cortes comerciais (pescoço, perna e paleta), excetuando-se o de costilhar, que cresceu linearmente. A espessura de gordura e a área de olho-de-lombo foram influenciadas pelos níveis de concentrado. Observou-se efeito significativo sobre os constituintes nãocarcaça, exceto o trato digestório, que não foi afetado pelos níveis de concentrado. O aumento do nível de concentrado influenciou o peso e o rendimento dos cortes e órgãos internos e aumentou a proporção e a deposição de gordura na carcaça.
\end{abstract}

Palavras-chave: dieta, gordura, órgãos, peso, rendimento

\section{Effect of concentrate levels on retail cuts, non-carcass and leg components of crossbred feedlot lambs}

\begin{abstract}
Thirty-two intact lambs averaging $17.1 \mathrm{~kg}$ of initial body weight were assigned to one of the following four treatments: $30,45,60$ or $75 \%$ of dietary concentrate in a completely randomized design (eight lambs/treatment). Lambs were feedlot fed during the experimental period (43 days). After slaughter, carcasses were refrigerated for $24 \mathrm{~h}$. It was measured the weights and percentages of five cuts from the half carcass as well as the proportion of muscle, bone and fat tissue from the leg. The weights of neck, shoulder, ribs, loin and leg all increased linearly by increasing the level of concentrate in the diet while the opposite was observed for the yields of neck, leg, and shoulder; yield of ribs followed the increment of concentrate in the diet and increased linearly. Fat thickness and loin eye area were both affected by the level of concentrate in the diet. The same was observed for the non-carcass components with the exception of digestive tract. Feeding increasing dietary levels of concentrate to lambs affected the weight and yield of retail cuts and internal organs and increased the proportion and deposition of fat in the carcass.
\end{abstract}

Key Words: diet, fat, organs, weight, yield

\section{Introdução}

A Região Nordeste tem se destacado pelo aumento da produção de carne ovina, possibilitado pelos cruzamentos industriais com raças especializadas. No entanto, o consumo da carne ovina ainda é muito baixo e está relacionado à qualidade do produto ofertado. Todavia, isso pode ser contornado quando há disponibilidade de um produto de 
qualidade no mercado e com oferta durante todo o ano. Para obtenção de maior fatia no mercado para a carne ovina, é preciso melhorar o sistema de produção. A maioria dos criadores coloca à venda animais com idade avançada com características de carcaça indesejáveis e baixo rendimento de sua porção comestível.

O sistema de produção e o nível nutricional dos animais que deles participam são fatores que interferem na qualidade das carcaças comercializadas. Esforços vêm sendo feitos com o objetivo de melhorar essas carcaças, entre eles é a tentativa de reduzir a idade de abate por meio do nível nutricional, visto que as diferentes proporções teciduais da carcaça são influenciadas por estes fatores. O crescimento relativo dos tecidos segue a lei da harmonia anatômica, obedecendo a seguinte ordem cronológica: osso, músculo e gordura (pélvico-renal e subcutânea), sendo que a deposição de gordura aumenta com a idade (Wood et al., 1980) e o tipo de alimentação dos cordeiros. Dietas ricas em concentrado determinam maior disponibilidade de energia e favorecem o crescimento do tecido adiposo, reduzindo o rendimento da porção comestível da carcaça e comprometendo sua comercialização (Sousa, 1993). É preciso estabelecer um nível de concentrado adequado no intuito de se obter maior quantidade de músculo e adequado acabamento (Figueró, 1979). Níveis superiores a $70 \%$ acarretam acúmulo de gordura e tendem a reduzir o rendimento dos cortes pelo incremento da quantidade de gordura depositada (Furusho-Garcia et al., 2004), desvalorizando, portanto, os cortes comerciais (Sainz, 1996). Entre os cortes da carcaça ovina, a perna é considerada o mais nobre, pois apresenta o maior acúmulo de massa muscular (Monteiro et al., 1999), daí a importância de sua composição tecidual para avaliação de sua qualidade (Neres et al., 2001).

Além da carcaça, determinados subprodutos aproveitáveis, conhecidos como quinto quarto ou componentes não-carcaça (Osório, 1996), compostos por órgãos e vísceras, também possuem valor comercial. No Nordeste do Brasil, os componentes não-carcaça, conhecidos como buchada, são comercializados em quilograma e constituem $30 \%$ do valor do animal, podendo ser utilizados como fonte adicional de renda, contribuindo para compensação dos custos de abate (Silva Sobrinho, 2001). O peso dos componentes não-carcaça varia de acordo com a porcentagem de energia consumida.

Neste trabalho, determinou-se a influência dos níveis de concentrado sobre o peso e o rendimento dos cortes e dos componentes não-carcaça e a composição tecidual da perna de cordeiros mestiços de Dorper terminados em confinamento ou mestiços de Dorper x Santa Inês.

\section{Material e Métodos}

O experimento foi realizado na Estação Experimental de Pendência, base física da Empresa Estadual de Pesquisa Agropecuária da Paraíba (EMEPA-PB), localizada em Soledade-PB. Foram utilizados 32 cordeiros mestiços ( $1 \frac{1}{2}$ Dorper x $1 / 2$ Santa Inês), machos não-castrados, na fase de terminação, com 90 dias de idade e peso médio inicial de $17,1 \mathrm{~kg}$. Os animais foram distribuídos em um delineamento inteiramente casualizado, com quatro tratamentos e oito repetições. Efetuou-se análise de regressão, pelo programa Statistical Analysis System (SAS, 1985), de todas as variáveis estudadas em função do nível de concentrado na dieta utilizada.

As dietas foram formuladas segundo o NRC (1985), com teores semelhantes de proteína ( $15 \%$ de PB com base na MS), sendo utilizados quatro níveis de concentrado (30, 45, 60 ou 75\%) e feno de tifton 85 (Cynodon dactylon) como volumoso. A ração com $30 \%$ de concentrado foi calculada para atender às exigências nutricionais dos animais, objetivando ganho de $250 \mathrm{~g} /$ dia. Na Tabela 1 consta a composição percentual e bromatológica das dietas utilizadas no ensaio.

As dietas foram oferecidas em duas refeições diárias, de modo a ocorrer aproximadamente $10 \%$ de sobras. As

Tabela 1 - Composições percentual e bromatológica das dietas, com base nas proporções de concentrado (em \% da MS)

Table 1 - Ingredient and chemical compositions of the experimental diets (\% of DM)

\begin{tabular}{|c|c|c|c|c|}
\hline & \multicolumn{4}{|c|}{$\begin{array}{c}\text { Nível de concentrado (\%) } \\
\text { Concentrate level }\end{array}$} \\
\hline & 30 & 45 & 60 & 75 \\
\hline Feno de tifton (Tifton hay) & 70,0 & 55,0 & 40,0 & 25,0 \\
\hline Farelo de milho (Corn meal) & 5,81 & 20,0 & 32,4 & 45,2 \\
\hline Farelo de soja (Soybean meal) & 9,00 & 10,0 & 12,0 & 13,5 \\
\hline Farelo de trigo (Wheat bran) & 5,00 & 6,01 & 8,00 & 10,0 \\
\hline Melaço (Molasses) & 2,00 & 2,00 & 2,00 & 2,00 \\
\hline Óleo de soja (Soybean oil) & 4,00 & 3,00 & 2,00 & 1,00 \\
\hline Núcleo mineral (Mineral mixture) ${ }^{1}$ & 2,00 & 2,00 & 2,00 & 2,00 \\
\hline Uréia (Urea) & 1,5 & 1,2 & 0,7 & 0,3 \\
\hline Calcáreo (Limestone) & 0,7 & 0,8 & 0,9 & 1,0 \\
\hline $\operatorname{MS}(D M)$ & 91,5 & 90,7 & 91,8 & 91,8 \\
\hline MO (OM) & 88,4 & 89,8 & 89,1 & 90,5 \\
\hline $\mathrm{PB}(C P)$ & 15,2 & 15,3 & 15,3 & 15,4 \\
\hline FDN (NDF) & 57,6 & 46,5 & 35,7 & 24,9 \\
\hline FDA $(A D F)$ & 28,6 & 22,8 & 17,3 & 11,2 \\
\hline $\mathrm{EE}(E E)$ & 5,50 & 4,80 & 4,20 & 3,50 \\
\hline EM (kcal/kgMS) (ME, kcal/kg DM) & 2,30 & 2,40 & 2,60 & 2,70 \\
\hline $\mathrm{Ca}$ & 1,13 & 1,13 & 1,17 & 1,12 \\
\hline $\mathrm{P}$ & 0,50 & 0,53 & 0,58 & 0,60 \\
\hline
\end{tabular}


quantidades oferecidas foram ajustadas por ocasião da pesagem dos animais, a cada 14 dias, sem jejum de sólidos e líquidos. O experimento teve duração total de 43 dias de confinamento, precedido de um período de adaptação de oito dias. No final do período experimental, todos os animais foram abatidos após jejum de sólidos ( 24 horas) e líquidos (16 horas). Após o abate, efetuou-se a evisceração com a
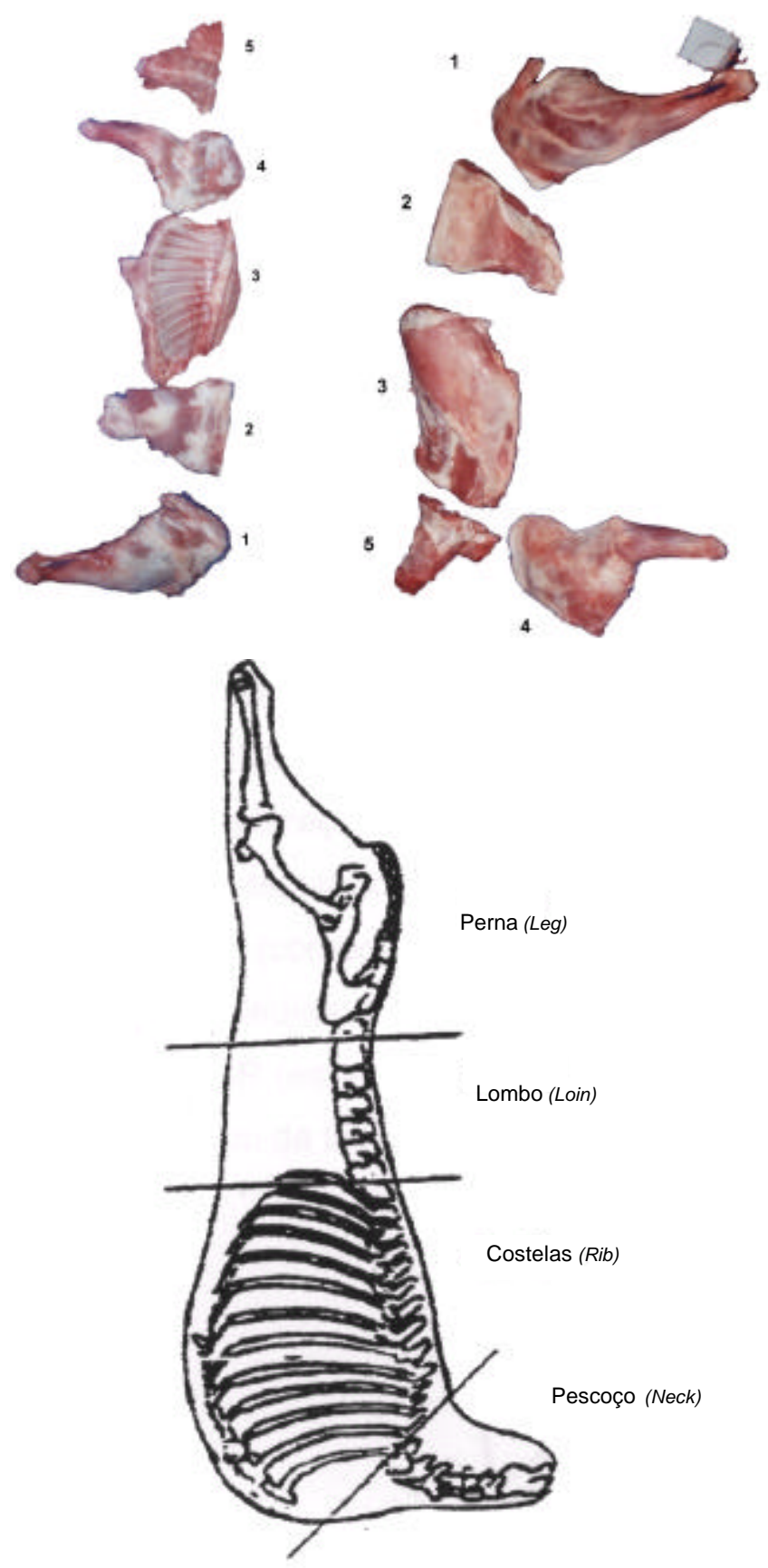

Figura 1 - Cortes comerciais da meia-carcaça esquerda de ovinos $1 / 2$ Dorper x Santa Inês (1 - perna, 2 - lombo, 3 - costelas, 4 - paleta, 5 - pescoço).

Figure 1 - Retail cuts of the left half carcass of $1 / 2$ Dorper $x 1 / 2$ Santa Inês lambs (1 - leg, 2 - loin, 3 - rib, 4 - shoulder, 5 - neck). separação dos órgãos brancos (rúmen-retículo, omaso, abomaso e intestinos) e vermelhos (coração, fígado, diafragma, rins, pulmão + brônquios), do sangue, da gordura visceral (omental e mesentérica) e de alguns componentes corporais externos, como cabeça, pés e couro. Após a separação, foram pesados para determinação de seus pesos individuais e obtenção do rendimento de buchada (RB \%) $=\left[\left(\sum \mathrm{OC}(\mathrm{kg}) / \mathrm{PA}(\mathrm{kg})\right] \mathrm{x} 100\right)$, em que OC (órgãos comestíveis = órgãos vermelhos e órgãos brancos) e PA = peso de abate. As carcaças foram transferidas para uma câmara frigorífica a $4^{\circ} \mathrm{C}$, onde foram mantidas por 24 horas. Posteriormente, foram pesadas e divididas longitudinalmente acompanhando a linha central da coluna vertebral. Em seguida, a meiacarcaça esquerda foi subdividida em paleta, perna, lombo e costilhar (Figura 1).

Após a pesagem dos cortes, a perna foi acondicionada em saco plástico e congelada para posterior dissecação. Foram separados músculos, ossos e gorduras subcutânea e intermuscular, que foram quantificados segundo procedimentos estabelecidos por McCutcheon et al. (1993). Foram determinados os pesos dos músculos que envolvem o fêmur (Semimembranosus, Semitendinosus, Bicepsfemoris, Quadriceps femoris, Aductor) e avaliadas as quantidades de gordura total, gordura intermuscular e gordura subcutânea, a relação gordura subcutânea:intermuscular, o peso de ossos e o comprimento do fêmur (CF) e, ainda, o índice de musculosidade (IM) da perna, obtido conforme descrito por Purchas et al. (1991), utilizando-se a fórmula $\mathrm{IM}=(\sqrt{\mathrm{PM} / \mathrm{CF}}) / \mathrm{CF}$, em que PM equivale ao somatório do peso (g) dos cinco músculos descritos anteriormente.

A determinação da área de olho-de-lombo foi realizada a partir de um corte transversal entre a $12^{\mathrm{a}}$ e a 13 a vértebra torácica, efetuando-se em transparência plástica o desenho da área, em correspondência à porção cranial do lombo, estabelecendo-se a largura e a profundidade máxima para o cálculo da área de olho-de-lombo (AOL), conforme sugerido por Silva Sobrinho et al. (2002), a partir da seguinte fórmula: $\mathrm{AOL}=(\mathrm{A} / 2 \times \mathrm{B} / 2) \pi$, em que: $\mathrm{A}=$ largura $\mathrm{B}=$ profundidade. Também foi determinada a espessura da gordura subcutânea (EGS), que é a espessura máxima de gordura de cobertura sobre a superfície da 13 a costela, a $11 \mathrm{~cm}$ da linha dorsolombar.

\section{Resultados e Discussão}

Conforme dados apresentados na Tabela 2, os pesos (g) de pescoço, paleta, costelas, lombo e perna tiveram efeito linear crescente à medida que se elevaram os níveis de concentrado na dieta, o que pode ser atribuído ao fato de a dieta não ser isoenergética. No caso dos cortes expressos 
Tabela 2 - Médias, coeficientes de variação (CV), equações de regressão (ER) e coeficientes de determinação $\left(r^{2}\right)$ para os cortes comerciais, em relação ao peso de carcaça fria (PCF), a espessura de gordura subcutânea e a área de olho-de-lombo (AOL) de cordeiros $1 / 2$ Dorper $\times$ Santa Inês alimentados com dietas formuladas com diferentes níveis de concentrado

Table 2 - Means, coefficients of variation (CV), regression equation (RE) and coefficient of determination $\left(r^{2}\right)$ of the retail cuts, expressed as proportion of cold carcass weight (CCW), fat thickness (FT) and loin eye area (LEA) on 1/2 Dorper $+1 / 2$ Santa Inês lambs fed increasing levels of concentrate in the diet

\begin{tabular}{|c|c|c|c|c|c|c|c|}
\hline & \multicolumn{4}{|c|}{$\begin{array}{c}\text { Nível de concentrado (\%) } \\
\text { Concentrate level }\end{array}$} & \multirow[t]{2}{*}{$\mathrm{CV}(\%)$} & \multirow[t]{2}{*}{$r^{2}$} & \multirow[t]{2}{*}{$\begin{array}{l}\mathrm{ER} \\
R E\end{array}$} \\
\hline & 30 & 45 & 60 & 75 & & & \\
\hline Pescoço (Neck) (g) & 388 & 477 & 499 & 650 & 22,56 & 0,89 & $\hat{\mathrm{Y}}=236,81+5,2542 \mathrm{x}^{*}$ \\
\hline Paleta (Shoulder) (g) & 815 & 961 & 972 & 1.128 & 17,39 & 0,98 & $\hat{\mathrm{Y}}=634,58+6,6173 \mathrm{x} *$ \\
\hline Costelas (Rib) (g) & 1.097 & 1.327 & 1.518 & 1.896 & 19,84 & 0,99 & $\hat{Y}=566,04+6,6173 x^{*}$ \\
\hline Lombo (Loin) (g) & 498 & 651 & 605 & 845 & 22,03 & 0,86 & $\hat{\mathrm{Y}}=301,88+6,7917 \mathrm{x}^{*}$ \\
\hline Perna (g) (Leg) & 1.485 & 1.795 & 1.840 & 2.060 & 17,78 & 0,94 & $\hat{\mathrm{Y}}=1186,7+12,117 \mathrm{x}^{*}$ \\
\hline Pescoço (\% PCF) (Neck, \% CCW) & 13,41 & 10,51 & 10,10 & 9,81 & 23,35 & 0,73 & $\hat{\mathrm{Y}}=14,662-0,0734 \mathrm{x} *$ \\
\hline Paleta (\% PCF) (Shoulder, \%CCW) & 18,46 & 17,78 & 17,51 & 16,69 & 4,92 & 0,95 & $\hat{\mathrm{Y}}=19,466-0,0361 \mathrm{x} *$ \\
\hline Costelas (\% PCF) (Rib, \%CCW) & 24,48 & 24,68 & 26,98 & 28,12 & 7,0 & 0,90 & $\hat{\mathrm{Y}}=21,694+0,085 \mathrm{x}^{*}$ \\
\hline Lombo (\% PCF) (Loin, \%CCW) & 11,16 & 11,97 & 10,81 & 12,61 & 11,02 & 0,90 & $\hat{\mathrm{Y}}=11,63$ \\
\hline Perna (\%PCF) (Leg, \% CCW) & 33,46 & 32,26 & 32,97 & 30,53 & 5,43 & 0,76 & $\hat{\mathrm{Y}}=35,87-0,0627 \mathrm{x}^{*}$ \\
\hline Espessura de gordura (mm) (Fat thickness) & 0,88 & 0,925 & 0,971 & 1,02 & 20,77 & 0,95 & $\hat{\mathrm{Y}}=0,7369+0,0038 \mathrm{x}$ \\
\hline $\mathrm{AOL}\left(\mathrm{cm}^{2}\right)(L E A)$ & 7,79 & 9,41 & 9,97 & 12,52 & 17,50 & 0,98 & $\hat{\mathrm{Y}}=4,7655+0,1008 \mathrm{x} *$ \\
\hline
\end{tabular}

* Significativo a $5 \%$ de probabilidade pelo teste $\mathrm{F}$.

* Significant at $5 \%$ of probability by F test.

em porcentagem do peso de carcaça fria, houve efeito linear decrescente $(\mathrm{P}<0,05)$ apenas para pescoço, paleta e perna. Como foram considerados cortes de desenvolvimento precoce, a maior disponibilidade de energia nos níveis mais elevados de concentrado pode ter contribuído para a redução nos seus percentuais de 0,0734; 0,0361 e 0,0627\%, respectivamente, para cada $1 \%$ da inclusão do concentrado na ração (Tabela 2). Os altos níveis de concentrado na ração (maior quantidade de energia disponível) contribuíram para o aumento do tecido adiposo nos pesos mais elevados e, com isso, ocorreu desvalorização destes cortes. A porcentagem de lombo, no entanto, não foi influenciada pelos níveis de concentrado utilizados, sendo obtido valor médio de $11,63 \mathrm{~kg}$. Os resultados deste estudo foram superiores aos descritos por Siqueira et al. (2001) e Alves et al. (2003).

Neste estudo, o maior percentual de costelas ou costilhar em relação aos demais cortes pode estar relacionado ao fato de esse corte ser de desenvolvimento mais tardio e os animais terem sido abatidos com 141 dias de idade (não haviam atingido, portanto, sua maturidade fisiológica). Provavelmente, a maior disponibilidade de nutrientes da dieta com maior nível de concentrado contribuiu para o maior desenvolvimento do tecido muscular em relação ao adiposo. Todavia, esses resultados não estão de acordo com os descritos por Alves et al. (2003), que avaliaram três níveis de EM $(2,42 ; 2,66$ e 2,83 mcal/kg) em dietas para ovinos Santa Inês e observaram elevação na porcentagem de costelas com a elevação do teor de energia na dieta.

À medida que se elevaram os níveis de concentrado, houve aumento na espessura de gordura subcutânea (EGS) (Tabela 2). Valores superiores aos obtidos nesta pesquisa foram constatados por Bueno et al. (2000), entre 1,2 e 1,4 mm em ovinos da raça Sulffock abatidos com $35 \mathrm{~kg}$, e Oliveira et al. (2003), que encontraram EGS de 2,74 e 3,24 mm, em cordeiros Santa Inês e Bergamácia com 53,4 e 52,8 kg de peso corporal, respectivamente. Como a área de olho-delombo (AOL) é considerada indicador de musculosidade do animal, observou-se, pela significância dos dados, que, com o acréscimo dos níveis de concentrado, houve efeito significativo linear crescente $(\mathrm{P}<0,05)$ para esta característica, indicando que nas maiores proporções de concentrado os cordeiros apresentaram maior musculosidade na carcaça. Valores inferiores foram constatados por Carvalho et al. (1980) em cordeiros das raças Corriedale, Ideal e Romney Marsh. Esses autores verificaram valores de $7,52 \mathrm{~cm}^{2}$ para a AOL, no entanto, Zundt et al. (2003) e Marques (2003) obtiveram valores superiores $\left(13,48\right.$ e $13,07 \mathrm{~cm}^{2}$, respectivamente) em cordeiros mestiços Ile de France e Bergamácia e tricross (Texel + Bergamácia + Corriedale) abatidos, respectivamente, com 30 e $40 \mathrm{~kg}$.

Verificou-se efeito significativo linear crescente $(\mathrm{P}<0,05)$ (Tabela 3$)$ sobre os componentes sangue, fígado, 
rins, brônquios + pulmões, baço e coração com o aumento do concentrado na dieta. O peso, em gramas, do componente sangue foi influenciado pelos níveis de concentrado na ração. Os maiores níveis favoreceram o crescimento dos cordeiros, que apresentaram maiores pesos de abate $(21,68$; 24,$03 ; 25,55$ e $27,30 \mathrm{~kg}$ nos níveis de 30, 45, 60 e 75\% de concentrado) e, conseqüentemente, maior aporte sangüíneo.

Estes resultados corroboram os relatos de Medeiros et al. (2003), que encontraram valores de 1.009; 1.006 e 0,910 g, em cordeiros Santa Inês com 32,0; 29,0 e 27,9 kg de PV. Os pesos de rúmen-retículo, omaso, abomaso e intestinos (delgado + grosso), na forma como foram expressos, não foram influenciados pelos níveis de concentrado na dieta, sendo obtidos valores de 582,26; 56,13; 126,10 e 1.059,35 kg, respectivamente.

Apesar de o coração e os pulmões serem órgãos que mantêm sua integridade e serem prioritários na utilização de nutrientes, independentemente do nível de alimentação (Ferreira et al., 2000), neste estudo, houve efeito da dieta sobre os pesos desses componentes, que aumentaram linearmente à medida que se elevou o nível de concentrado na ração (Tabela 3 ).

Os pesos de fígado, baço e rins também aumentaram linearmente em resposta à elevação dos níveis de concentrado (Tabela 3) na dieta. Como são órgãos com elevadas taxas metabólicas, relacionadas à dieta, o peso elevado do fígado pode ser atribuído à maior proporção de concentrado na dieta. Esses resultados não estão de acordo com os encontrados por Alves et al. (2003). No entanto, Siqueira et al. (2001), em experimento com cordeiros mestiços Ile de France $\times$ Corriedale forneceram dietas contendo 2,4 mcal/ $\mathrm{kg}$ MS e observaram valores de 0,520 e 0,180 kg para fígado e coração, respectivamente, superior ao deste estudo, com nível similar de energia.

Houve efeito linear positivo $(\mathrm{P}<0,05)$ dos níveis de concentrado sobre o tamanho de cabeça e pés, sendo estimados incrementos de 8,336 e $2,521 \mathrm{~cm}$ para cada percentual de concentrado na dieta (Tabela 3 ), que podem

Tabela 3 - Peso médio ( $\mathrm{g}$ ), coeficiente de variação (CV), equação de regressão (ER) e coeficiente de determinação $\left(\mathrm{r}^{2}\right)$ para os componentes não-carcaça de cordeiros $1 / 2$ Dorper x $1 / 2$ Santa Inês alimentados com dietas formuladas com diferentes níveis de concentrado

Table 3 - Means, coefficient of variation (CV), regression equation (RE) and coefficient of determination $\left(r^{2}\right)$ of the non-carcass components on $1 / 2$ Dorper $x \frac{1}{2}$ Santa Inês lambs fed increasing levels of concentrate in the diet

\begin{tabular}{|c|c|c|c|c|c|c|c|}
\hline & \multicolumn{4}{|c|}{$\begin{array}{c}\text { Nível de concentrado (\%) } \\
\text { Concentrate level }\end{array}$} & \multirow[t]{2}{*}{$\mathrm{CV}(\%)$} & \multirow[t]{2}{*}{$\mathrm{r}^{2}$} & \multirow[t]{2}{*}{$\begin{array}{l}\mathrm{ER} \\
R E\end{array}$} \\
\hline & 30 & 45 & 60 & 75 & & & \\
\hline Coração (Heart) & 100,0 & 105,0 & 108,1 & 116,4 & 14,0 & 0,96 & $\hat{\mathrm{Y}}=89,04+0,349 \mathrm{x}^{*}$ \\
\hline Fígado (Liver) & 815 & 961 & 972 & 1.128 & 17,4 & 0,82 & $\hat{Y}=634,58+6,6173 x^{*}$ \\
\hline Rins (Kidney) & 1.097 & 1.327 & 1.518 & 1.896 & 19,8 & 0,95 & $\hat{Y}=566,04+6,6173 x^{*}$ \\
\hline Baço (Spleen) & 498 & 651 & 605 & 845 & 22,0 & 0,95 & $\hat{\mathrm{Y}}=301,88+6,7917 \mathrm{x} *$ \\
\hline Pulmão+brônquios (Lung) & 1.485 & 1.795 & 1.840 & 2.060 & 17,8 & 0,99 & $\hat{\mathrm{Y}}=1186,7+12,117 \mathrm{x}^{*}$ \\
\hline Sangue (Blood) & 762,5 & 801,2 & 835,6 & 1.039 & 16,9 & 0,86 & $\hat{\mathrm{Y}}=557,0+5,764 \mathrm{x}^{*}$ \\
\hline Rúmen/retículo (Rumen/reticulum) & 566,2 & 565,6 & 573,0 & 630,0 & 14,2 & 0,69 & $\hat{\mathrm{Y}}=582,26$ \\
\hline Omaso (Omasum) & 56,20 & 57,50 & 50,01 & 61,4 & 24,1 & 0,82 & $\hat{\mathrm{Y}}=56,13$ \\
\hline Abomaso (Abomasum) & 126,2 & 123,7 & 135,6 & 120,0 & 19,6 & 0,84 & $\hat{\mathrm{Y}}=126,1$ \\
\hline Intestinos (Intestine) & 1.024 & 1.057 & 1.053 & 1.110 & 11,5 & 0,90 & $\hat{\mathrm{Y}}=1059,35$ \\
\hline Cabeça (Head) & 1.394 & 1.509 & 1.654 & 1.762 & 11,9 & 0,99 & $\hat{\mathrm{Y}}=1142,3+8,336 \mathrm{x} *$ \\
\hline Pés (Feet) & 560,6 & 663,7 & 658,1 & 688,6 & 14,2 & 0,84 & $\hat{\mathrm{Y}}=510,4+2,5214 \mathrm{x}^{*}$ \\
\hline Gordura omental (Omental fat) & 216,2 & 256,2 & 317,5 & 512,1 & 12,3 & 0,68 & $\hat{\mathrm{Y}}=626,0+0,6326 \mathrm{x}^{*}$ \\
\hline Gordura mesentérica (Mesenteric fat) & 170,6 & 196,8 & 208,1 & 264,2 & 13,1 & 0,72 & $\hat{\mathrm{Y}}=302,52+1,948 \mathrm{x} *$ \\
\hline Buchada* & 3173 & 2499 & 3462 & 4319 & 13,0 & 0,88 & $\hat{\mathrm{Y}}=2454,6+22,84 \mathrm{x} *$ \\
\hline Rendimento da buchada (\%) (Buchada yield) & 15,10 & 14,80 & 14,20 & 15,90 & 8,9 & & $\hat{\mathrm{Y}}=19,890 \mathrm{x}+0,002 \mathrm{x}^{2}$ \\
\hline Panelada** (g) & 5.111 & 5.663 & 5.704 & 6.770 & 11,9 & & $\hat{\mathrm{Y}}=4107,3+33,70 \mathrm{x}^{*}$ \\
\hline Rendimento da panelada (\%) (Panelada yield) & 24,4 & 23,9 & 23,54 & 24,4 & 24,9 & & $\hat{\mathrm{Y}}=24,06$ \\
\hline
\end{tabular}

* Buchada - prato tradicional da culinária nordestina. Normalmente, fazem parte de sua composição coração, pulmões, fígado, intestinos, rins, rúmen e o sangue (Traditional dish of the Brazilian Northeastern cookery; It includes by-products such as heart, lungs, liver, intestines, kidneys, rumen and blood).

* Panelada - prato tradicional da culinária nordestina. Normalmente, fazem parte de sua composição os constituintes da buchada + pés e cabeça (Traditional dish of the Brazilian Northeastern cooker; it includes the ingredients used in the buchada plus feet and head). 
estar relacionados ao maior desenvolvimento dos animais, decorrente da maior disponibilidade de nutrientes nos tratamentos com maiores níveis de concentrado.

Neste estudo, houve efeito significativo linear crescente $(\mathrm{P}<0,05)$ para as gorduras visceral, omental e mesentérica (Tabela 3). O aumento do concentrado na dieta proporcionou maior quantidade de gordura depositada. Este resultado corrobora os de Kosloski (2002), que afirmou que o aumento do concentrado promove ruminalmente aumento na concentração de ácido propiônico e diminuição na relação acetato:propionato, resultando em maior disponibilidade de energia (glicose circulante), o que favorece a secreção de insulina e induz a lipogênese, aumentando a deposição de gordura.

O aumento dos níveis de concentrado afetou significativamente $(\mathrm{P}<0,05)$ o peso da buchada, que aumentou conforme elevaram os níveis de concentrado. No entanto, houve efeito quadrático no rendimento e sua derivação ajustou a um ponto de máxima no nível de $70 \%$ de concentrado, no qual manteve o melhor rendimento. Medeiros et al. (2003) obtiveram valores superiores aos encontrados neste trabalho (4,76 a 5,62 kg). Santos (2003), no entanto, encontrou rendimento de buchada de 19,24 e $18,84 \%$ em cabritos alimentados com dietas contendo 55 e $40 \%$ de concentrado, respectivamente.
Os níveis de concentrado tiveram efeito linear positivo $(\mathrm{P}<0,05)$ sobre o peso da panelada (buchada + cabeça + pés $)$ expresso em gramas. Não foi verificado, entretanto, efeito significativo $(\mathrm{P}>0,05)$ da proporção de concentrado sobre o rendimento da panelada, obtendo-se valor médio de $24,06 \%$.

Na Tabela 4 constam os pesos médios, em gramas, obtidos para os tecidos muscular, adiposo e ósseo da perna, o comprimento e a circunferência do fêmur, as relações músculo:gordura e músculo:osso e a musculosidade da perna. O nível de concentrado na dieta afetou todos os tecidos musculares da perna. Influenciou ainda, de forma linear crescente $(\mathrm{P}>0,05)$, o comprimento e a circunferência do fêmur e as gorduras subcutânea e intermuscular, o que pode ser explicado pelo aumento do nível de concentrado na ração, que provavelmente provocou mudança na fermentação ruminal ocasionando diferenciação no perfil dos ácidos graxos voláteis (AGVs) produzidos no rúmen e disponibilizando maior quantidade de ácido propiônico em relação ao ácido acético, que contribuiu para o aumento da energia disponível e favoreceu a maior porcentagem de gordura subcutânea e intermuscular. Com o aumento de $1 \%$ no nível de concentrado, houve acréscimo de 1,482 e $0,801 \mathrm{~kg}$ das gorduras subcutânea e intermuscular, respectivamente.

Tabela 4 - Médias e equações de regressão dos pesos dos componentes da perna de cordeiros $1 \frac{1}{2}$ Dorper $\times$ Santa Inês alimentados com dietas formuladas com diferentes níveis de concentrado

Table 4 - Means and regression equations (RE) of the weights of leg components on $1 / 2$ Dorper $+1 / 2$ Santa Inês lambs fed increasing levels of concentrate in the diet

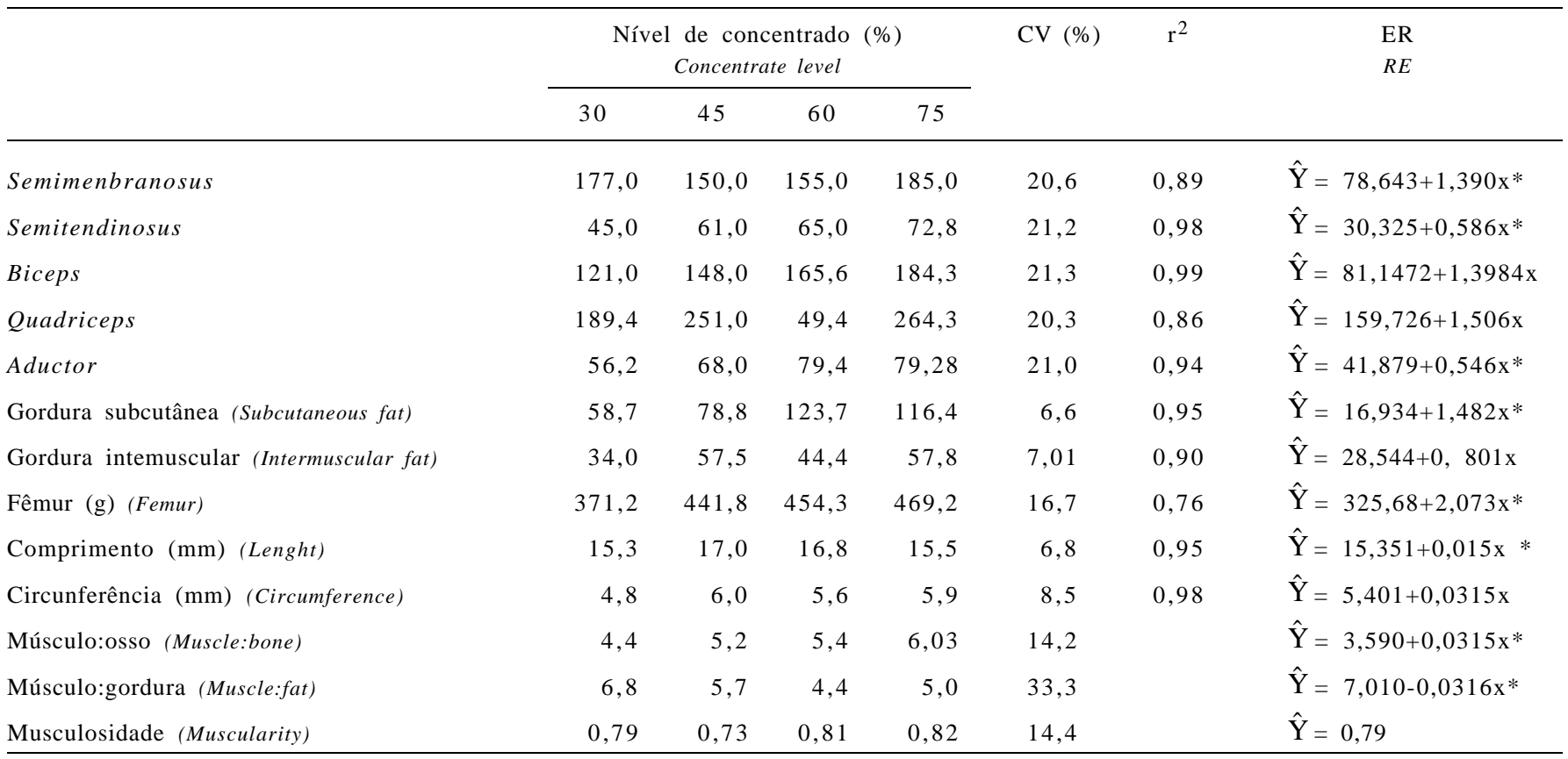

* Significativo a $5 \%$ de probabilidade pelo teste $\mathrm{F}$.

* Significant at $5 \%$ of probability by F test. 
A relação músculo:osso $(\mathrm{M}: \mathrm{O})$ apresentou comportamento significativo linear crescente $(\mathrm{P}<0,05)$, indicando que à medida que aumentou a proporção de concentrado na dieta ocorreu aumento na quantidade de músculo em relação à de ossos na carcaça, o que está de acordo com os resultados obtidos por Pereira et al. (2004) e Silva Sobrinho et al. (2002), que verificaram maior relação M:O nos animais que receberam maior quantidade de concentrado. Para a relação músculo:gordura (M:G), ocorreu o inverso: nos tratamentos com maior proporção de concentrado, verificou-se aumento na quantidade de gordura. O tecido ósseo é o que possui crescimento mais precoce, sendo seguido do muscular e, por último, do adiposo (Wood et al., 1980). Segundo Sainz (1996), o tecido adiposo apresenta crescimento mais acentuado em animais mais velhos. Neste estudo, apesar de os animais não terem atingido sua maturidade fisiológica por completo, apresentaram aumento no conteúdo de gordura na carcaça conforme elevou o nível de concentrado na ração.

\section{Conclusões}

O aumento dos níveis de concentrado resultou em aumento no peso e em decréscimo nos rendimentos dos cortes comerciais. O corte costilhar, provavelmente em virtude de maior precocidade, apresentou maior rendimento em relação aos demais.

Os níveis de concentrado estudados afetaram características como espessura de gordura e gordura visceral, indicando que estas variáveis apresentam valores mais elevados quanto maior a adição de concentrado à dieta.

Maiores níveis de concentrado proporcionaram aumento dos constituintes teciduais, especialmente de músculos e gordura, devendo-se avaliar a implicação do seu uso na qualidade da carne de ovinos mestiços de Santa Inês.

\section{Literatura Citada}

ALVES, K.S.; CARVALHO, F.F.R.; FERREIRA, M.A. et al. Níveis de energia em dietas para ovinos Santa Inês: características de carcaças e constituintes corporais. Revista Brasileira de Zootecnia, v.32, p.1927-1936, 2003.

BUENO, M.S.; CUNHA, E.A.; SANTOS, L.G. et al. Características de carcaça de cordeiros Suffolk abatidos em diferentes idades. Revista Brasileira de Zootecnia, v.29, p.1803-1810, 2000.

CARVALHO, J.B.P.; PEDROSO, J.R.; FIGUERÓ, P.R.P. et al. Alguns fatores que afetam o rendimento da carne ovina. Revista Centro de Ciências Rurais, v.10, p.95-104, 1980.

FERREIRA, M.A.; VALADARES FILHO, S.C; MUNIZ, E.B.H. et al. Características das carcaças, biometria do trato gastrintestinal, tamanho dos órgãos internos e conteúdo gastrintestinal de bovino ps F1 Simental x Nelore alimentados com vários níveis de concentrados. Revista Brasileira de Zootecnia, v.29, n.4, p.1174-1182, 2000.
FIGUERÓ P.R.P. Rendimento de carcaça em ovinos no Rio Grande do Sul. In: JORNADA TÉCNICA DE PRODUÇÃO OVINA NO RIO GRANDE DO SUL, 1979, Bajé. Anais... Bajé: EMBRAPAEMATER RS-ARCO-SAGRI, 1979. p.65-78.

FURUSHO-GARCIA, I.F.; PÉREZ, J.R.O.; BONAGURIO, A.L.M et al. Estudo dos cortes da carcaça de cordeiros Santa Inês puros e cruzas Santa Inês com Texel, Ile de France e Bergamácia. Revista Brasileira Zootecnia, v.33, p.453462,2004

KOSLOSKI, G.V. Bioquímica dos ruminantes. Santa Maria: Universidade Federal de Santa Maria, 2002. 140p.

MARQUES, C.A.T. Desempenho e características de carcaça de cordeiros criados com acesso a comedouros seletivos (creep feeding) e terminados em confinamento. Jaboticabal: Universidade Estadual Paulista, 2003. 68p. Dissertação (Mestrado em Zootecnia) - Universidade Estadual Paulista, 2003.

McCUTCHEON, S.N.; BLAIR, H.T.; PURCHAS, R.W. Body composition and organ weights in fleeceweight- selected and control Romney rams. New Zealand Journal of Agriculture Research, v.36, p.445-449, 1993.

MEDEIROS, A.N.; HONÓRIO, A.F.; LISBOA, A.C.C. et al. Desempenho de cordeiros Santa Inês alimentados com farelo de Girassol. In: REUNIÃO ANUAL DA SOCIEDADE BRASILEIRA DE ZOOTECNIA, 40., 2003, Santa Maria. Anais... Santa Maria: Sociedade Brasileira de Zootecnia, 2003. (CD-ROM).

MONTEIRO, A.L.G.; GARCIA, C.A.; NERES, M.A. et al. Pesos e rendimentos dos cortes e órgãos de cordeiros confinados alimentados com polpa cítrica. Revista Unimar Ciências, v.8, p.97-100, 1999 .

NATIONAL RESEARCH COUNCIL - NRC. Nutrient requirements of sheep. 6.ed. Washington, D.C.: National Academy of Science, 1985. 99p.

NERES, M.A.; MONTEIRO, A.L.G.; GARCIA, C.A. et al. Forma física da ração e pesos de abate nas características de carcaça de cordeiros em creep feeding. Revista Brasileira de Zootecnia, v.30, p.948-954, 2001.

OLIVEIRA, M.V.M.; PERÉZ, J.R.O.; FURUSHO-GARCIA, I.F. et al. Desempenho de cordeiros das raças Bergamácia e Santa Inês, terminados em confinamento, recebendo dejetos de suínos como parte da dieta. Revista Brasileira de Zootecnia, v.32, p.1391-1396, 2003.

OSÓRIO, M.T.M. Estudio Comparativo de la cualidad de la canal y de la carne em rasa Aragonesa, Ojinegra de Teruel y. Bibilitana. Zaragoza: Universidade de Zaragoza, 1996. 199p. Tese (Doutorado em Veterinària) - Universidade de Zaragosa, 1996.

PEREIRA, V.O.; GONZAGA NETO, S.; MEDEIROS, A.N. et al. Caracterização centesimal da carne de ovinos Santa Inês alimentados com feno de Maniçoba (Manihot pseudoglaziovii Pax Hoffman). In: CONGRESSO NORDESTINO DE PROdUÇÃo Animal, 3., 2004, Campina Grande. Anais... Campina Grande: Sociedade Nordestina de Produção Animal, 2004. (CD-ROM).

PURCHAS, R.W.; DAVIES, A.S.; ABDULlAH, A.Y. et al. An objective measure of muscularity: changes with animal growth and differents between genetics lines of Southdown sheep. Meat Science, v.30, p.81-94, 1991.

SAINZ, R.D. Qualidade das carcaças e da carne ovina e caprina In: REUNIÃO ANUAL DA SOCIEDADE BRASILEIRA DE ZOOTECNIA, SEMANA DE CAPRINOCULTURA E OVINOCULTURA TROPICAL BRASILEIRA, 2., 1996. Fortaleza. Anais... Fortaleza: Sociedade Brasileira de Zootecnia, 1996. p.3-14.

SANTOS, I.B. Utilização do feno de capim-elefante em rações completas para caprinos leiteiros em recria. Areia: Universidade Federal da Paraíba, 2003. 69p. Dissertação (Mestrado em Zootecnia) - Universidade Federal da Paraíba, 2003. 
STATISTICAL ANALYSIS SYSTEM - SAS. User's guide statistics. Cary: 1985. 956p.

SILVA SOBRINHO, A.G. Criação de ovinos. 2.ed. Jaboticabal: FUNEP, 2001. 302p.

SILVA SOBRINHO, A.G.; MACHADO, M.R.F.; GASTALDI, K.A. et al. Efeitos da relação volumoso:concentrado e do peso ao abate sobre os componentes da perna de cordeiros Ile de France x Ideal confinados. Revista Brasileira de Zootecnia, v.31, p.1017-1023, 2002.

SIQUEIRA, E.R.; SIMÕES, C.D.; FERNANDES, S. Efeito do sexo e do peso ao abate sobre a produção de carne de cordeiro. Morfometria da carcaça, pesos dos cortes, composição tecidual e componentes não constituintes da carcaça. Revista Brasileira de Zootecnia, v.30, p.1299-1307, 2001.

SOUSA, O.C.R. Rendimento de carcaça, composição regional e física da paleta e quarto em cordeiros Romney Marsh abatidos aos 90 e 180 dias de idade. Pelotas: Universidade Federal de Pelotas, 1993. 120p. Dissertação (Mestrado em Zootecnia) - Universidade Federal de Pelotas, 1993.
WOOD, J.D.; MACFIE, H.J.H.; POMEROY, R.W. et al. Carcass composition in four sheep breeds: the importance of type of breed and stage of maturity. Animal production, v.30, p.135152,1980 .

ZUNDT, M.; MACEDO, F.F.; MARTINS, E.N. et al. Características de carcaça de cordeiros terminados em confinamento, com dietas contendo diferentes níveis protéicos. Ciência Rural, v.33, n.3, p.565-571, 2003 . 